\title{
Optimization Analysis of Adaptive UKF Filtering Algorithm in Self Alignment of SINS
}

\section{Wan-xin Su${ }^{1}$}

Changchun Institute of Optics, Fine Mechanics and Physics, Chinese Academy of Sciences, Changchun, 130033, China

E-mail:ccswx@163.com

In SINS, the inertial components are directly mounted on the carrier.The error can be divided into deterministic error and random drift error (dynamic error), in which, the formercan be compensated. In this case, the initial alignment of the pure static base can achieve a high accuracy. In the practical application, the dynamic error is directly reflected in the inertial device because of influence of the external environment (wind, vibration and disturbance, etc.). At this time, the error model is not linear. Under this situation, unscented Kalman filter (UKF) and adaptive unscented Kalman filter (AUKF) were designed respectively. We introduced the principle of adaptive estimation into the original UKF algorithm, adjusted the contribution of the kinetic model to navigation solution. AUKF algorithm can automatically balance the weight ratio of state and observation information in filtering to adjust the covariance of state vector and observation vector in real-time, thereby to improve the system performance. Experimental results showed that the use of adaptive UKF algorithm in comparion with the normal UKF algorithm can obtain better accuracy and reliability of self alignment.

ISCC 2015

18-19, December 2015

Guangzhou, China

\section{${ }^{1}$ Speaker}




\section{Introduction}

Before the SINS work, the calculated navigation coordinate system needs to accurately align and track the real geographic coordinate system. The initial alignment is to establish the strapdown matrix as a benchmark for the inertial navigation. The initial alignment requirements are fast and accurate. As the initial alignment of SINS is a digital platform based on computer, the algorithm error of the strapdown system is very significant. Because of direct installation of the inertial components on the carrier, the disturbance (such as vibration, wind and load changes, etc.) of the external environment has significantly affected the inertial device, which results in a dynamic error.

Under the condition of estimation criterion, according to a certain statistical meaning, the optimal estimation is achieved. There are different estimation methods as to different estimation criteria. For the initial self alignment of static SINS, Kalman optimal filter is commonly used in the linear discrete system. The estimated value is a linear function of the observed values and the covariance of the estimation error is minimized.

If the error model is nonlinear, the nonlinear filtering is needed.The method of nonlinear self alignment is determined [1]. KF, EKF and UKF filtering algorithms are compared. The results show that the KF filter is no longer suitable to the nonlinear filtering, and the effect of UKF is better than EKF. The implementation of UKF in self alignment of SINS is studied [2].

In this paper, the unscented Kalman filter (UKF) and the improved adaptive UKF (AUKF) are designed and compared for the initial self alignment of the static base SINS. The divergence degree together with the aiming accuracy of the filter are optimized as well.

\section{Establishing State and Observation Equation}

\subsection{Coarse Alignment Process}

The self alignment is divided into coarse alignmentand fine alignment[3], in which, the task of the former is to quickly obtain a rough initial attitude matrix. The coarse alignment is usually referred to as the analytical alignment. On the coarse alignment stage, depend on the observation value of the acceleration of gravity $g$ and the Earth's rotation angular velocity

$\omega_{i e}$, and the attitude matrix from the carrier coordinate system to the navigation system is directly estimated.

In the initial alignment of the static base, we chose the carrier's navigation coordinate system (n) for (E,N,U) geographic coordinate system (t). The component of the acceleration $g$ vector was measured by accelerometer in the carrier coordinate system (b). Similarly, the component of the the Earth's rotation angular velocity $\omega_{i e}$ was measured by gyroscope.

In case of local latitude, the component of the gravity vector and the earth self rotation angular velocity vector are known in the geographic coordinate system as below:

$$
\begin{aligned}
& g^{n}=\left[\begin{array}{ll}
0 & 0-g
\end{array}\right]^{T} \\
& \omega_{i e}^{n}=\left[\begin{array}{ll}
0 & \omega_{i e}^{n} \cos L \\
\omega_{i e}^{n} \sin L
\end{array}\right]
\end{aligned}
$$

The conversion relationship between the carrier coordinate system and the navigation coordinate system is as in Eq. (2.2): 


$$
\begin{aligned}
& g^{b}=C_{n}^{b} g^{n} \\
& \omega_{i e}^{b}=C_{n}^{b} \omega_{i e}^{n}
\end{aligned}
$$

Because $C_{n}^{b}$ is orthogonal, it is satisfied with $C_{b}^{n}=\left(C_{n}^{b}\right)^{-1}=\left(C_{n}^{b}\right)^{T}$.

By vector multiplication, we got the other 3 vectors: $g \times \omega_{i e}, g \times \omega_{i e} \times g, \omega_{i e} \times g \times \omega_{i e}$ with two methods to discuss [4]. Upon analysis of the coarse alignment, the obtained attitude matrix was rectfied by the misalignment angle and became more accurately. The correction procedure is as shown below:

$$
\dot{C}_{b}^{n}=(I-\phi) C_{b}^{n}
$$

Here, $\mathrm{I}$ is the unit matrix. $\phi$ is the correction matrix of the misalignment angle.

$$
\phi=\left[\begin{array}{ccc}
0 & -\phi_{U} & \phi_{E} \\
\phi_{U} & 0 & -\phi_{N} \\
-\phi_{E} & \phi_{N} & 0
\end{array}\right]
$$

The first method is shown below:

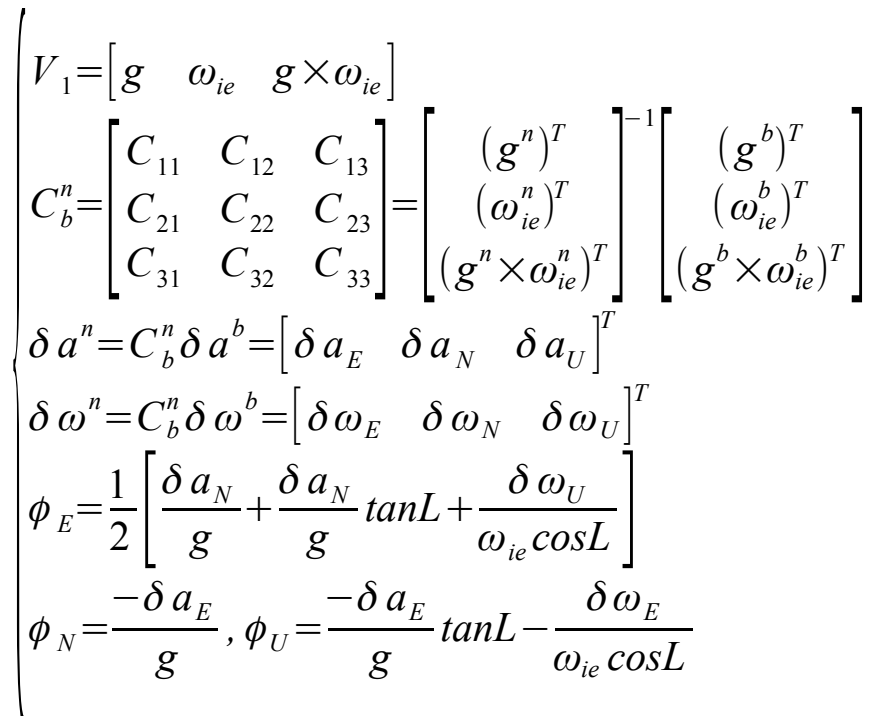

The second Method is as follows:

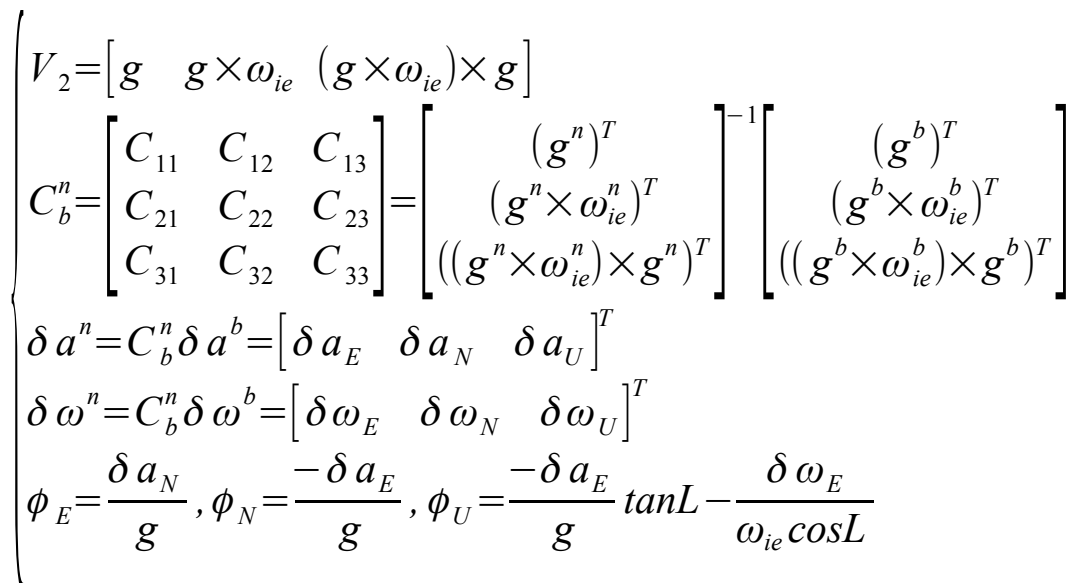

In comparison of both methods, the results show that the east misalignment angle in the second is not affected by the output error of the up gyro and accelerometer. In the strapdown inertial sensors, the precision of the accelerometer is much higher than that of the gyroscope; 
besides, the output error of the up gyro is an important factor to influence the east misalignment angle. Based on the above reasons, the second method is better than the first.

\subsection{Establishing State and Observation Equation}

After coarse alignment, the initial attitude matrix is established. The goal of the fine alignment is to update the attitude matrix $C_{b}^{n}$ to make sure that the navigation system is gradually close to a real geographic coordinate system.

For nonlinear filtering algorithm, the state and observation equations are firstly established. The state variables and variables of the system are as follows[5]:

$$
\begin{aligned}
& X=\left[\delta \dot{V}_{E}, \delta \dot{V}_{N}, \dot{\phi}_{E}, \dot{\phi_{N}}, \dot{\phi}_{U}, \nabla_{x}, \nabla_{y}, \varepsilon_{x}, \varepsilon_{y}, \varepsilon_{z}\right]^{T} \\
& Z=\left[\delta \dot{V}_{E}, \delta \dot{V}_{N}\right]^{T}
\end{aligned}
$$

The observation value is the horizontal velocity error $\delta \dot{V}_{E}, \delta \dot{V}_{N}$ in the navigation coordinate system; $\dot{\phi_{E}}, \dot{\phi_{N}}, \dot{\phi_{U}}$ are misalignment angles. $\nabla_{x}, \nabla_{y}$ are random constant bias of horizontal accelerometer in the carrier coordinate system; $\varepsilon_{x}, \varepsilon_{y}, \varepsilon_{z}$ are random constant drift of gyroscope in three directions of the system.

The nonlinear state and the observation equation of the fine alignment of the SINS system[6] is in Eq.(2.8):

$$
\begin{aligned}
& X=A X+f(x)+G W \\
& Z=H X+V=\left[\begin{array}{ll}
I_{2 \times 2} & 0_{2 \times 8}
\end{array}\right] X+V
\end{aligned}
$$

The state equation is divided into the linear part $\mathrm{AX}$ and the nonlinear part $f(x)$. Among them:

$$
\begin{aligned}
& A=\left[\begin{array}{cccccccccc}
0 & 2 \Omega_{U} & 0 & -g & 0 & C_{11} & C_{12} & 0 & 0 & 0 \\
-2 \Omega_{U} & 0 & g & 0 & 0 & C_{21} & C_{22} & 0 & 0 & 0 \\
0 & \frac{-1}{R_{M}} & 0 & \Omega_{U} & -\Omega_{E} & 0 & 0 & C_{11} & C_{12} & C_{13} \\
\frac{1}{R_{N}} & 0 & -\Omega_{U} & 0 & 0 & 0 & 0 & C_{21} & C_{22} & C_{23} \\
\frac{\tan L}{R_{N}} & 0 & \Omega_{E} & 0 & 0 & 0 & 0 & C_{31} & C_{32} & C_{33}
\end{array}\right] \\
& f(x)=\left[\begin{array}{l}
0_{2 \times 1} \\
-\Omega_{N} \sin \phi_{U} \\
\Omega_{N}\left(1-\cos \phi_{U}\right) \\
\Omega_{N}\left(-\phi_{E} \sin \phi_{U}+\phi_{N} \cos \phi_{U}\right) \\
0_{5 \times 1}
\end{array}\right], \quad A=\left[\begin{array}{ccccc}
C_{11} & C_{12} & 0 & 0 & 0 \\
C_{21} & C_{22} & 0 & 0 & 0 \\
0 & 0 & C_{11} & C_{12} & C_{13} \\
0 & 0 & C_{21} & C_{22} & C_{23} \\
0 & 0 & C_{31} & C_{32} & C_{33} \\
& & 0_{5 \times 5} &
\end{array}\right]
\end{aligned}
$$




$$
\Omega_{U}=\omega_{i e} \sin L, \Omega_{N}=\omega_{i e} \cos L . W=\left[\begin{array}{llllll}
w_{\delta V_{E}^{n}} & w_{\delta V_{N}^{n}} & w_{\phi x} & w_{\phi y} & w_{\phi z} & 0_{1 \times 5}
\end{array}\right]^{T} \text { is }
$$

zero mean Gaussian white noise of the system. $\quad V=\left[\begin{array}{lll}w_{\delta V_{E}} & w_{\delta V_{N}}\end{array}\right]^{T}$ refers to the zero mean measurement of Gaussian white noise. $\mathrm{L}$ is the local latitude.

\subsection{Establishing Error Model}

Considering the fine alignment time is short, gyroscopes and accelerometers error model are both approximately considered as constant drift with white noise: $\nabla=\nabla_{b}+\omega_{a}, \varepsilon=\varepsilon_{b}+\omega_{g}$

$\nabla_{b}=0, \quad w_{a}$ is the Gaussian white noise of accelerometers; $\varepsilon_{b}=0, \quad w_{g}$ is gaussian white noise of gyroscopes. $\dot{\phi_{E}}, \dot{\phi_{N}}, \dot{\phi_{U}}$

Generally, for the horizontal misalignment angle $\phi_{E}$ and $\phi_{N}$ which are small after coarse alignment, $\sin \phi_{E}$ and $\sin \phi_{N}$ are approximately equal to $\phi_{E}$ and $\phi_{N}$. $\cos \phi_{E}$ and $\cos \phi_{N}$ are approximately close to 1.; however, the azimut misalignment angle is too large to be estimated. The initial alignment of the static base on the earth surface is:

$$
f_{E}=f_{N} \approx 0, f_{U}=-g
$$

The error equation of IMU inertial navigation system is shown as below:

$$
\left\{\begin{array}{l}
\delta \dot{V}_{E}=-g\left(\phi_{N} \cos \phi_{U}+\phi_{E} \sin \phi_{U}\right)+2 \omega_{i e}(\sin L) \delta V_{N}+\nabla_{E} \\
\delta \dot{V}_{N}=-g\left(\phi_{N} \sin \phi_{U}-\phi_{E} \cos \phi_{U}\right)-2 \omega_{i e}(\sin L) \delta V_{E}+\nabla_{N} \\
\dot{\phi_{E}}=-\sin \phi_{U} \omega_{i e} \cos L+\phi_{N} \omega_{i e} \sin L-\delta V_{N} / R_{m}+\varepsilon_{E} \\
\dot{\phi_{N}}=\left(1-\cos \phi_{U}\right) \omega_{i e} \cos L-\phi_{E} \omega_{i e} \sin L+\delta V_{E} / R_{n}+\varepsilon_{N} \\
\dot{\phi_{U}}=\left(-\phi_{N} \sin \phi_{U}+\phi_{E} \cos \phi_{U}\right) \omega_{i e} \cos L+\delta V_{E} \tan L / R_{n}+\varepsilon_{U}
\end{array}\right.
$$

$\omega_{i e}$ is the angular velocity of the Earth's rotation; $\varepsilon_{E}, \varepsilon_{N}, \varepsilon_{U}$ show gyro error of the navigation coordinate system; $\mathrm{L}$ is the geographic latitude; $\mathrm{h}$ is the geographic height; $R_{m}$ is the radius of curvature of meridian; $R_{n}$ is the prime vertical radius of curvature. $\nabla_{E}$ and $\nabla_{N}$ are the horizontal error of the accelerometer in the navigation coordinate system.

\section{UKF Algorithm and AUKF Algorithm}

\subsection{UKF Algorithm}

In order to improve the effect of nonlinear filtering, Julier proposed an unscented Kalman filter (Unscented Kalman Filter, UKF) method to estimate the nonlinear filtering problem. First, this method processes Unscented Transform (U tranformation) on the state equation, estimates the filtering result after the UT and in turn reduces the estimation error[7]. Unlike the Extended Kalman Filter (EKF) to linearize the nonlinear system model or calculte the Jacobian matrix. UKF deals directly with the nonlinear model, which becomes increasingly widely-used in the navigation system.

For one step estimate equation, UKF uses the assured sample strategy directly to handle nonlinear deliver of the system's mean value and covariance error. The design step of UKF is described as below.

Initialize: 


$$
\left\{\begin{array}{l}
\hat{x}_{0}=E\left[x_{0}\right] \\
P_{0}=E\left[\left(x_{0}-\hat{x}_{0}\right)\left(x_{0}-\hat{x}_{0}\right)^{T}\right]
\end{array}\right.
$$

Calculate theSigma Point and Corresponding Weight:

$$
\begin{cases}x_{0, k-1}=x_{\hat{k-1}} & \\ x_{i, k-1}=x_{k-1}^{\hat{n}}+\sqrt{(n+\lambda) P_{k-1}} & ,(i=1,2, \ldots, n) \\ x_{i, k-1}=x_{k-1}-\sqrt{(n+\lambda) P_{k-1}} & ,(i=n+1, n+2, \ldots, 2 n) \\ w_{0}^{(m)}=\lambda /(n+\lambda) & \\ w_{0}^{(c)}=\lambda /(n+\lambda)+\left(1-\zeta^{2}+\eta\right) & \\ w_{i}^{(m)}=w_{i}^{(c)}=1 /(2(n+\lambda)) & ,(i=1,2, \ldots, 2 n)\end{cases}
$$

$\lambda=\zeta^{2}(n+\kappa)-n$ is a scaling factor; $\zeta$ can determine the value range of sampling points, which is usually chosen between a small positive number between $0-1$, such as $1 \mathrm{e}-3 ; \kappa$ is a constant, generally 0 or $3-n ; \eta$ depends on distribution of state variables. $\eta=2$ is optimal when $\mathrm{x}$ is subject to Gauss distribution. $w_{i}^{(m)}$ and $w_{i}^{(c)}$ are the weights of the mean value and variance.

Update thetime:

$$
\left\{\begin{array}{l}
x_{i, k / k-1}=f\left(x_{i, k-1}\right) \\
\hat{\bar{x}}_{k}=\sum_{n=0}^{2 \mathrm{n}} w_{i}^{(m)} x_{i, k / k-1} \\
\bar{P}_{k}=\sum_{n=0}^{2 \mathrm{n}} w_{i}^{(c)}\left[x_{i, k / k-1}-\hat{\bar{x}}_{k}\right]\left[x_{i, k / k-1}-\hat{\bar{x}}_{k}\right]^{T}+Q_{k} \\
Z_{i, k / k-1}=H \times x_{i, k / k-1} \\
\hat{\bar{Z}}_{k}=\sum_{n=0}^{2 \mathrm{n}} w_{i}^{(m)} Z_{i, k / k-1}
\end{array}\right.
$$

$Q_{k}$ is the variance of the system noise w at k moment.

Updation measurement:

$$
\left\{\begin{array}{l}
P_{Z_{k}, Z_{k}}=\sum_{n=0}^{2 n} w_{i}^{(c)}\left[Z_{i, k / k-1}-\hat{\bar{Z}}_{k}\right]\left[Z_{i, k l k-1}-\hat{\bar{Z}}_{k}\right]^{T}+R_{k} \\
P_{x_{k}, Z_{k}}=\sum_{n=0}^{2 n} w_{i}^{(c)}\left[x_{i, k / k-1}-\hat{\bar{x}}_{k}\right]\left[Z_{i, k / k-1}-\hat{\bar{Z}}_{k}\right]^{T} \\
K_{k}=P_{x_{k}, Z_{k}} \times P_{Z_{k}, Z_{k}}^{-1} \\
\hat{x}_{k}=\hat{\bar{x}}_{k}+K_{k}\left(Z_{k}-\hat{\bar{Z}}_{k}\right) \\
P_{k}=\bar{P}_{k}-K_{k} \times P_{Z_{k}, Z_{k}} \times K_{k}^{T}
\end{array}\right.
$$


$Z_{k}$ is the measurement vector at $k$ moment; $R_{k}$ is the variance of the measurement at $k$ moment. By analyzing the calculation steps of UKF, it's assumed that the method have the following advantages.

The calculation accuracy of nonlinear Gaussian distribution statistics achieves at least the third order in comparison with the second order for the non-Gaussian distribution;

There is no need to make a clear understanding of the specific form of the nonlinear function, derivatives and Jacobians matrix;

The whole complexity of the algorithm equals to EKF, but UKF can lessen the influence of truncation error of the higher-order terms and is easy to be implemented.

UKF filtering method for estimating the nonlinear filtering problem not only provides the above unique advantages, but also overcomes a series of problems available in EKF[8]. In practice, however, it's discovered that UKF is more sensitive to the choice of the initial value. An error in the initial value will directly affect the outcome of filtering result; moreover, even if the initial value is reasonable, the existence of various disturbance errors and uncertainty of statistical noise models will also affect the accuracy of UKF filtering. This paper, based on the principle of adaptive algorithm, puts forward a brand new filter estimation method to achieve fine alignment.

\subsection{AUKF Algorithm}

The adaptive UKF algorithm and the traditional UKF have basically the same structure[9]. Some adjustments are performed as in Eq.(3.5):

$$
\left\{\begin{array}{l}
P_{Z_{k}, Z_{k}}=\frac{1}{a_{k}} \sum_{n=0}^{2 \mathrm{n}} w_{i}^{(c)}\left[Z_{i, k / k-1}-\hat{\bar{Z}}_{k}\right]\left[Z_{i, k / k-1}-\hat{\bar{Z}}_{k}\right]^{T}+R_{k} \\
P_{x_{k}, Z_{k}}=\frac{1}{a_{k}} \sum_{n=0}^{2 \mathrm{n}} w_{i}^{(c)}\left[x_{i, k / k-1}-\hat{\bar{x}}_{k}\right]\left[Z_{i, k / k-1}-\hat{\bar{Z}}_{k}\right]^{T} \\
P_{k}=\frac{1}{a_{k}} \bar{P}_{k}-K_{k} \times P_{Z_{k}, Z_{k}} \times K_{k}^{T}
\end{array}\right.
$$

$a_{k}$ is the adaptive factor, the initial value is taken as 1 and the value range $0 \leq a_{k} \leq 1$. If the value of $a_{k}$ is reasonable, it is possible to balance the ratio between the prediction and measurement information in the system model. $a_{k}$ is constructed as in Eq. (3.6):

$$
a_{k}= \begin{cases}1 & \operatorname{tr}\left(V_{k} V_{k}^{T}\right) \leq \operatorname{tr}(P) \\ \frac{\operatorname{tr}(P)}{\operatorname{tr}\left(V_{k} V_{k}^{T}\right)} & \operatorname{tr}\left(V_{k} V_{k}^{T}\right)>\operatorname{tr}(P)\end{cases}
$$

Taking a prediction residual, covariance matrix is:

$$
P=\sum_{n=0}^{2 \mathrm{n}} w_{i}^{(c)}\left[Z_{i, k / k-1}-\hat{\bar{Z}}_{k}\right]\left[Z_{i, k / k-1}-\hat{\bar{Z}}_{k}\right]^{T}
$$

The above impoved UKF algorithm shows that when there is bias in the initial value selection or abnormal disturbance in system model, $a_{k}$ will be less than 1 , indicating the weight of forecast information in system model is as low as possible in the final filtered result. 
When the prediction of the system model is obviously abnormal, $a_{k}$ will approach 0 . That is to say, the system model forecast information should be abandoned. Obviously, $a_{k}$ is able to adaptively adjust $\quad \hat{\bar{x}}_{k}$ by the predicted residual $\mathrm{V}_{\mathrm{k}}$ and the measurement information $\hat{\bar{Z}}_{k}$.

\section{Experimental Analysis}

The initial parameters were set as below: Latitude $\mathrm{L}=43.50^{\circ}$; the initial value of the state variable $\mathrm{x}$ is 0 ; the initial misalignment angles after coarse alignment are $\left[0.324^{\circ} 0.358^{\circ} 3.31^{\circ}\right]$; gyro constant drift $1 \% \mathrm{~h}$, random drift $0.5^{\circ} / \mathrm{h}$; the initial bias of accelerometer at three directions are $0.5 \mathrm{mg}$, random deviations is $0.1 \mathrm{mg}$; horizontal velocity error is $0.1 \mathrm{~m} / \mathrm{s}$. Experimental results are shown in Fig.e 1, Fig. 2, Fig. 3.

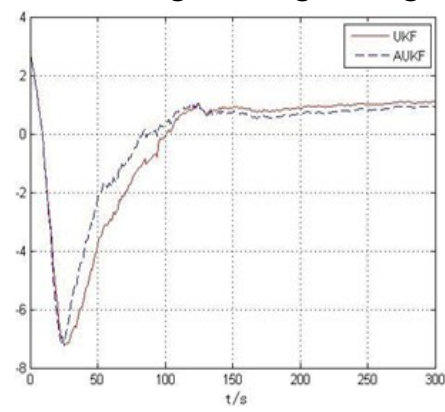

Figure 1 : $\Phi_{\mathrm{E}}$ angle

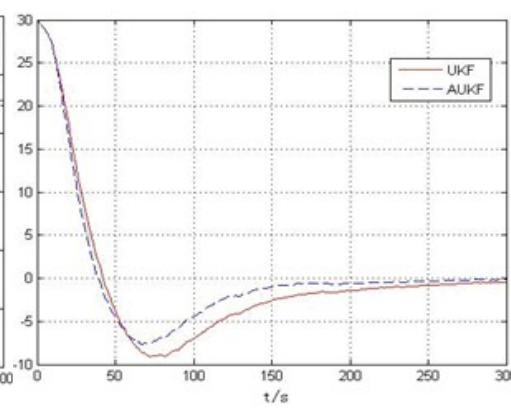

Figure 2: $\Phi_{\mathrm{N}}$ angle

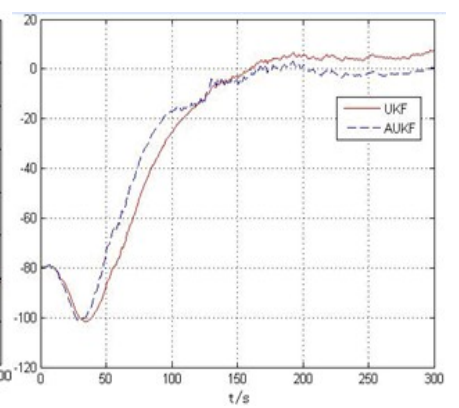

Figure 3: $\Phi_{\mathrm{U}}$ angle

The figures show that the convergence precision of three misalignment angles are $1.2^{\prime}, 0.3^{\prime}$ and 6.0' when using the conventional UKF filtering algorithm. The convergence precision of adaptive UKF (AUKF) are improved by $1.0^{\prime}, 0.1^{\prime}$ and $1.0^{\prime}$. In alignment, the convergence time of the AUKF's three misalignment angles were 110s, 152s and 173s compared to $120 \mathrm{~s}, 180 \mathrm{~s}$ and 200s of UKF respectively.

\section{Conclusion}

For the initial self alignment of the static base SINS, the accuracy and convergence rate of the misalignment angles have been improved when using the AUKF algorithm compared with UKF in all three directions: East, North and Up. The accuracy was increased by $0.2^{\prime}, 0.2^{\prime}$ and $5.0^{\prime}$; the convergence time was shortened by $10 \mathrm{~s}, 28 \mathrm{~s}$ and $27 \mathrm{~s}$ respectively because when the AUKF filter is disturbed by the initial value error and the system model, the covariance of the state vector and the observation vector can be adjusted in real time by the weight ratio of the state and observation information in the filtering results.

\section{References}

[1] L.Y. Shen. Key Technologies Research on Independent Alignment of Strap-Down Inertial Navigation Syetem[D]. Harbin Institute of Technology, June, 2014.

[2] B.C. Zhou,X.H. Cheng.Robust UKF algorithm in SINS initial alignment[J]. Journal of Southeast University ( English Edition),2011,27(1):56-65.

[3] C.S. Zhao, Y.Y. Qin, et al. A gravity-based anti-interference coarse alignment algorithm[J]. J of Astronautics, 2010, 31(10): 2335-2339. 
[4] W.H. Chai , X.R. Shen . Error Analysis of Analytic Rough Alignment for Marine SDINS[J]. Journal of Harbin Engineering University, Vol. 20(4), 1999:46-50.

[5] W.X. Su, C.M. Huang, P.W. Liu, et al. Application of adaptive Kalman filter technique in initial alignment of inertial navigation system[J]. Journal of Chinese Inertial Technology, 2010,18(1):4447.

[6] T. Yuan, Z.G. Qu. Algorithm Research on Independent Alignment of Aircraft Carrier's SINS[J]. Journal of Projectiles, Rockets, Missiles and Guidance, 2013,Vol.33(2):24-30.

[7] D.J. Wan,J.C. Fang . Initial alignment of inertial navigation[M], Southeast University Press,1998.

[8] C.M. Huang,X.H. Sun. Two algorithms for auto alignment in rocking base of SINS[J], Journal of Chang chun University of Technology(Natural Science Edition, 2013,Vol.34(6):711-714.

[9] H.D. Hu, X.L. Huang. SINS/CNS/GPS integrated navigation algorithm based on UKF[J]. Journal of Systems Engineering and Electronics,2010,21(1):102-109. 